Relations industrielles

Industrial Relations

\title{
Borgeat, Louis, La sécurité d'emploi dans le secteur public, Essai
}

\section{Renaud Paquet}

Volume 52, numéro 1, 1997

URI : https://id.erudit.org/iderudit/051159ar

DOI : https://doi.org/10.7202/051159ar

Aller au sommaire du numéro

\section{Éditeur(s)}

Département des relations industrielles de l'Université Laval

ISSN

0034-379X (imprimé)

1703-8138 (numérique)

Découvrir la revue

Citer ce compte rendu

Paquet, R. (1997). Compte rendu de [Borgeat, Louis, La sécurité d'emploi dans le secteur public, Essai]. Relations industrielles / Industrial Relations, 52(1),

212-214. https://doi.org/10.7202/051159ar

Tous droits réservés (C) Département des relations industrielles de l'Université Laval, 1997
Ce document est protégé par la loi sur le droit d'auteur. L'utilisation des services d'Érudit (y compris la reproduction) est assujettie à sa politique d'utilisation que vous pouvez consulter en ligne.

https://apropos.erudit.org/fr/usagers/politique-dutilisation/ 


\section{Recensions}

\section{Book Reviews}

\section{La sécurité d'emploi dans le secteur public, Essai}

par Louis BORGEAT, Sainte-Foy: Presses de l'Université du Québec, 1996, 135 p., ISBN 2-7605-0914-1.

Dans cet essai, l'auteur dresse un bilan de l'évolution du concept de la sécurité d'emploi dans le secteur public, concept qu'il qualifie, en avant-propos, de contrainte de gestion. Ce bilan, présenté sous une perspective manageriale, est très bien documenté. Même si le point de vue de l'administrateur public y prime, les nombreux éléments factuels et les arguments sont exposés de façon honnête. De par le sujet qu'il traite, cet ouvrage est unique. Autant le chercheur, l'étudiant ou le praticien des relations industrielles intéressé par les grandes problématiques du secteur public y trouvera son compte. L'Essai de Louis Borgeat contribuera certes à alimenter la réflexion et le débat sur la question de la sécurité d'emploi dans le secteur public.

L'auteur identifie deux volets bien distincts au concept de la sécurité d'emploi dans le secteur public. Il ne remet pas en question le premier volet qu'il qualifie de "politique". Ce dernier qui est utilisé pour désigner la permanence ou l'emploi pour une période indéterminée avait été consacré lors des réformes législatives des années 1960 afin de mettre fin au régime de patronage et aux importants mouvements de main-d'œuvre entourant les changements de gouvernement. À une époque où il était devenu important de rehausser la "qualité " de la fonction publique, le concept de permanence servait aussi de point d'appui à l'organisation de la carrière de l'employé de l'État.
C'est plutôt le deuxième volet de la sécurité d'emploi, soit le volet "économique ", que Louis Borgeat remet en question. La sécurité d'emploi "économique ", c'est celle qui a été négociée par les syndicats, d'abord de fonctionnaires, puis d'enseignants et de personnels de la santé et du réseau des affaires sociales. Elle donne à son titulaire une protection absolue qui empêche son employeur de le mettre à pied même si ce dernier n'a plus de travail pour lui. Seuls des motifs ayant trait au comportement de l'employé, à sa compétence ou à sa capacité d'exécuter le travail pourront alors être invoqués pour le congédier. Ces garanties d'emploi de nature économique furent accordées à une époque où l'employeur public n'envisageait aucunement de comprimer ses effectifs. Elles représentaient au plus une contrainte de gestion.

Après avoir brossé en première partie un tableau historique bien documenté de l'évolution des volets politique et économique du concept de la sécurité d'emploi dans le secteur public, l'auteur en présente en deuxième partie une remise en question. Cette dernière repose sur trois arguments: une situation politique qui a changée ; une comparaison avec les autres provinces canadiennes et avec le gouvernement fédéral ; un environnement économique différent.

La sécurité d'emploi n'est plus nécessaire pour protéger la neutralité de la 
fonction publique et pour éviter le népotisme. L'auteur rappelle qu'en plus du contexte et d'une culture politique qui est relativement intolérante face au favoritisme, les différentes lois et conventions collectives, outre les mesures de sécurité d'emploi, offrent des protections pour un traitement équitable lors de l'embauche et après l'embauche. Selon lui, le motif politique de la sécurité d'emploi ne tient plus.

La sécurité d'emploi dans le secteur public québécois ne résiste pas non plus à la comparaison canadienne. L'auteur présente tout d'abord les règles en vigueur au gouvernement fédéral. La sécurité d'emploi n'y est ni conventionnée, ni légiférée. Elle est plutôt l'objet d'une directive qui a été adoptée par le Conseil du trésor fédéral suite à des négociations avec les syndicats de fonctionnaires. Cette directive fournit une forme de sécurité d'emploi économique qui a cependant été suspendue en 1995 pour les employés des ministères où le Trésor fédéral prévoyait procéder aux coupures les plus importantes. L'ouvrage recense aussi les systèmes de sécurité d'emploi des autres provinces canadiennes et en arrive à conclure que le système québécois est nettement le plus généreux pour les employés de l'État.

Viennent ensuite les arguments conjoncturels spécifiques au contexte économique difficile. Les pressions concurrentielles obligent tant les entreprises que leur gouvernement à un maximum d'efficacité. À juste titre, l'auteur avance que les remises en question qui résultent de ces changements conjoncturels créent une grande turbulence dans l'organisation des services publics. Il faut pouvoir s'adapter rapidement ce qui signifie parfois de réduire la taille des effectifs de certains services. Or, selon l'auteur, la sécurité d'emploi a pour effet de limiter les possibilités d'ajustements de l'effectif. En ce sens, elle est un frein à l'adaptabilité de l'État et elle agit à contre-courant dans l'évolution que doit suivre l'administration de notre société.
Enfin, les difficultés économiques et la précarité qui caractérisent le statut d'emploi d'une grande partie des salariés du secteur privé dans les années 1990 contrastent avec la sécurité d'emploi des salariés du secteur public. N'y a-t-il pas là, souligne l'auteur, une préoccupation sur le plan de l'équité sociale lorsque ce sont ces mêmes salariés précaires qui contribuent à payer le coût de la garantie d'emploi des salariés du secteur public ? En fait, la notion de comparabilité de la rémunération entre les secteurs public et privé devrait aussi s'étendre à la sécurité d'emploi.

En guise de conclusion, l'auteur propose de maintenir le concept de permanence d'emploi, concept qui vise à garantir la stabilité des emplois mais remet en question celui de la sécurité d'emploi. Ainsi, l'employé pourrait occuper un poste dans le secteur public aussi longtemps qu'on ne met pas un terme à son engagement pour des motifs d'ordre disciplinaire ou professionnel, comme c'est le cas sous le régime actuel, ou pour des motifs d'ordre économique par le licenciement pour manque de travail selon la proposition formulée par l'auteur. Par contre, le licenciement pour manque de travail serait utilisé comme dernier recours car la sécurité d'emploi deviendrait un objectif à atteindre pour l'employeur respectueux de ses ressources humaines. Au dire de l'auteur, cette règle statique qu'est la sécurité d'emploi deviendrait alors une préoccupation dynamique qui ne pourrait que stimuler tous les acteurs.

Compte tenu de sa nature, il est difficile de présenter un résumé-critique de l'ouvrage de Louis Borgeat sans se prononcer sur les positions qu'il formule, ces dernières et la logique qui les soutient en occupant plus de la moitié de l'espace. Nous le ferons cependant en tentant d'être nous aussi le plus objectif possible en face de cette réalité qui laisse peu d'observateurs indifférents.

Tout d'abord, il est important de placer cette problématique à son vrai niveau en 
démystifiant quelque peu le concept de la sécurité d'emploi dans le secteur public. Alors que dans la fonction publique la proportion d'employés au statut précaire qui ne bénéficiaient pas de la sécurité d'emploi oscillait autour de $20 \%$ en 1995 , le taux de précarité double presque dans certains établissements du réseau de la santé ou de l'éducation. Compte tenu de ces données, il devient quelque peu difficile de soutenir l'argument à l'effet que les administrateurs de l'État québécois ne peuvent procéder aux adaptations nécessaires de la force de travail lorsqu'ils ont la capacité manageriale de se départir du cinquième, du quart ou du tiers de leurs effectifs sur simple non renouvellement de contrat. La réalité est que la sécurité d'emploi peut rendre les ajustements plus complexes. Elle peut aussi les ralentir dans certains cas. Par contre, il est important de mentionner qu'elle contribuera aussi à faciliter les ajustements en rassurant les employés affectés par les changements.

À une époque où la précarité occupe un espace toujours plus grand sur le marché du travail, le questionnement que propose Louis Borgeat tombe à point et est tout à fait pertinent d'autant plus qu'il repose sur une logique historique solide et des arguments bien articulés. Depuis près de deux décennies, il s'est développé dans notre société un consensus à l'effet qu'il devait exister une parité entre la rémunération et les conditions de travail des employés du secteur public et ceux du secteur privé. Il est clair que, même avec la précision que nous apportons à l'égard du fort taux de précarité, les employés du secteur public bénéficient d'un net avantage au chapitre de la sécurité d'emploi sur leurs concitoyens du secteur privé. Faut-il pour autant éliminer la sécurité d'emploi en s'assurant que si un groupe vit l'insécu- rité l'autre doit le faire ? Qu'y gagnerionsnous comme société?

Au niveau des comparaisons publicprivé, le secteur public syndiqué a pris un net recul sur le secteur privé syndiqué. En effet, les dernières études de l'Institut de recherche et d'information sur la rémunération démontrent que cet écart dépasserait les $6 \%$ à l'avantage du secteur privé syndiqué. Même s'il n'est pas possible de chiffrer la valeur monétaire de la sécurité d'emploi, nous ne croyons pas que cette dernière dépasse les $6 \%$. De prétendre autrement serait de croire que les dispositions actuelles régissant la sécurité d'emploi empêchent, à tout le moins, l'abolition de $6 \%$ des 500000 postes du secteur public et parapublic, soit 30000 postes.

Nous croyons au maintien de la sécurité d'emploi dans le secteur public. Cette dernière doit cependant être accompagnée d'assouplissements importants au niveau des règles qui encadrent les mouvements de personnel et l'organisation du travail dans le secteur public. Ces assouplissements dont la négociation a d'ailleurs commencé dans certains soussecteurs du secteur public, réduiraient grandement les coûts associés à l'administration de la sécurité d'emploi.

Même si nous ne partageons pas les conclusions de l'auteur, nous suggérons la lecture de cet ouvrage de Louis Borgeat à toute personne qui s'intéresse à la gestion des ressources humaines et aux relations du travail dans le secteur public. L'ouvrage est très bien écrit, agréable à lire et bien documenté. Il sera tout aussi utile pour l'enseignement universitaire qu'il le sera pour le praticien à la recherche d'arguments reposant sur une réflexion bien articulée.

RENAUD PAQUET

Université du Québec à Hull 